\title{
Temperature-dependent transport properties of FeRh
}

\author{
S. Mankovsky, S. Polesya, K. Chadova, and H. Ebert \\ Department Chemie, Ludwig-Maximilians-Universität München, 81377 München, Germany
}

J. B. Staunton

Department of Physics, University of Warwick, Coventry, United Kingdom

T. Gruenbaum, M. A. W. Schoen, and C. H. Back

Department of Physics, Regensburg University, Regensburg, Germany

X. Z. Chen and C. Song

Key Laboratory of Advanced Materials (MOE), School of Materials Science and Engineering, Tsinghua University, Beijing 100084, China

(Received 9 June 2016; revised manuscript received 16 March 2017; published 25 April 2017)

\begin{abstract}
The finite-temperature transport properties of FeRh compounds are investigated by first-principles densityfunctional-theory-based calculations. The focus is on the behavior of the longitudinal resistivity with rising temperature, which exhibits an abrupt decrease at the metamagnetic transition point, $T=T_{m}$, between ferroand antiferromagnetic phases. A detailed electronic structure investigation for $T \geqslant 0 \mathrm{~K}$ explains this feature and demonstrates the important role of (i) the difference of the electronic structure at the Fermi level between the two magnetically ordered states and (ii) the different degree of thermally induced magnetic disorder in the vicinity of $T_{m}$, giving different contributions to the resistivity. To support these conclusions, we also describe the temperature dependence of the spin-orbit-induced anomalous Hall resistivity and Gilbert damping parameter. For the various response quantities considered, the impact of thermal lattice vibrations and spin fluctuations on their temperature dependence is investigated in detail. Comparison with corresponding experimental data shows, in general, very good agreement.
\end{abstract}

DOI: 10.1103/PhysRevB.95.155139

\section{INTRODUCTION}

For a long time the ordered equiatomic FeRh alloy has attracted much attention owing to its intriguing temperaturedependent magnetic and magnetotransport properties. The crux of these features of this $\mathrm{CsCl}$-structured material is the first-order transition from an antiferromagnetic (AFM) to ferromagnetic (FM) state when the temperature is increased above $T_{m}=320 \mathrm{~K}[1,2]$. In this context the drop in the electrical resistivity that is observed across the metamagnetic transition is of central interest. Furthermore, if the AFM to FM transition is induced by an applied magnetic field, a pronounced magnetoresistance (MR) effect is found experimentally with a measured MR ratio of $\sim 50 \%$ at room temperature [2-4]. The temperature of the metamagnetic transition as well as the MR ratio can be tuned by the addition of small amounts of impurities [2,5-8]. These properties make FeRh-based materials very attractive for future applications in data storage devices. The origin of the large MR effect in FeRh, however, is still under debate. Suzuki et al. [9] suggest that, for deposited thin FeRh films, the main mechanism stems from the spin-dependent scattering of conducting electrons on localized magnetic moments associated with partially occupied electronic $d$ states [10] at grain boundaries. Kobayashi et al. [11] have also discussed the MR effect in the bulk ordered FeRh system, attributing its origin to the modification of the Fermi surface across the metamagnetic transition. So far only one theoretical investigation of the MR effect in FeRh has been carried out on an ab initio level [12].

\section{COMPUTATIONAL DETAILS}

The present study is based on spin-polarized electronic structure calculations using the fully relativistic multiple scattering Korringa-Kohn-Rostoker (KKR) Green's function method $[13,14]$ with the framework of spin density functional theory. For the self-consistent calculations a parametrization for the exchange and correlation potential based on the general gradient approximation (GGA) [15] has been used. For the charge and potential representation the atomic sphere approximation (ASA) has been applied. For the wave functions and corresponding matrices of the KKR formalism the cutoff value $l_{\max }=3$ has been used for the angular momentum expansion.

The central advantage of the KKR formalism is that it gives direct access to the retarded single-particle Green's function $G^{+}\left(\vec{r}, \vec{r}^{\prime}, E\right)$, which is given by [16-18]

$$
\begin{aligned}
G^{+}\left(\vec{r}, \vec{r}^{\prime}, E\right)= & \sum_{\Lambda \Lambda^{\prime}} Z_{\Lambda}^{m}(\vec{r}, E) \tau_{\Lambda \Lambda^{\prime}}^{m n}(E) Z_{\Lambda^{\prime}}^{n \times}\left(\vec{r}^{\prime}, E\right) \\
& -\delta_{m n} \sum_{\Lambda} Z_{\Lambda}^{n}(\vec{r}, E) J_{\Lambda}^{n \times}\left(\vec{r}^{\prime}, E\right) \Theta\left(r_{n}^{\prime}-r_{n}\right) \\
& +J_{\Lambda}^{n}(\vec{r}, E) Z_{\Lambda}^{n \times}\left(\vec{r}^{\prime}, E\right) \Theta\left(r_{n}-r_{n}^{\prime}\right)
\end{aligned}
$$

where the spatial vectors $\vec{r}$ and $\vec{r}^{\prime}$ are assumed to be within the atomic cell centered at sites $\vec{R}_{m}, \vec{R}_{n}$, respectively. Within the fully relativistic formulation used here the combined quantum number $\Lambda=(\kappa, \mu)$ stands for the relativistic spin-orbit and magnetic quantum numbers $\kappa$ and $\mu$, respectively [19]. 
Accordingly, $Z_{\Lambda}^{n}$ and $J_{\Lambda}^{n}$ are four-component wave functions obtained as regular and irregular solutions to the single-site Dirac equation for the isolated potential well $V^{n}$ centered at site $n$, respectively. The symbol " $\times$ " as a superscript of $Z_{\Lambda}^{n}$ and $J_{\Lambda}^{n}$ indicates the left-hand-side solution to the Dirac equation. Dealing with a magnetically ordered system within the framework of spin density functional theory, the potential $V^{n}$ is spin dependent. As a consequence $Z_{\Lambda}^{n}=\Sigma_{\Lambda^{\prime}} Z_{\Lambda^{\prime} \Lambda}^{n}$ (and also $J_{\Lambda}^{n}$ ) stands for a superposition of various partial waves with spin-angular character $\Lambda^{\prime}[20,21]$. Finally, the quantity $\tau_{\Lambda \Lambda^{\prime}}^{n n^{\prime}}$ is the so-called scattering path operator that represents the transfer of a wave coming in at site $n^{\prime}$ with character $\Lambda^{\prime}$ to a wave outgoing from site $n$ with character $\Lambda$ and all possible scattering events taking place in between [17].

The scheme sketched above to calculate the retarded Green's function gives direct access to the density of states (DOS) $n(E)$ via the expression

$$
n(E)=-\frac{\operatorname{Im}}{\pi} \int\left\langle G^{+}(\vec{r}, \vec{r}, E)\right\rangle_{c} d^{3} r .
$$

Information on the electronic structure more detailed than that given by the DOS is given by the Bloch spectral function (BSF) $A_{B}(\vec{k}, E)$. In terms of the retarded Green's function, this quantity is defined via

$$
\begin{aligned}
A_{B}(\vec{k}, E)= & -\frac{\operatorname{Im}}{\pi} \sum_{n, m} \exp \left[\imath \vec{k} \cdot\left(\vec{R}_{n}-\vec{R}_{m}\right)\right] \\
& \times \int\left\langle G^{+}\left(\vec{r}+\vec{R}_{n}, \vec{r}+\vec{R}_{m}, E\right)\right\rangle_{c} d^{3} r,
\end{aligned}
$$

where again the angular brackets specify an appropriate configurational average. For a perfectly ordered system the BSF would be a set of Dirac delta functions, $A_{B}(\vec{k}, E)=$ $\sum_{\gamma} \delta\left(E-E_{\vec{k}_{\gamma}}\right)$, and for $E=E_{F}$ it would trace out the Fermi surface. For a system with thermally induced spin fluctuations and lattice displacements the BSF has features with finite width from which the mean-free-path length of the electrons can be inferred.

The present approach used for the electronic structure calculations allows us to calculate the transport properties at finite temperatures on the basis of the linear response formalism using the Kubo-Středa expression for the conductivity tensor [22,23],

$$
\begin{aligned}
\sigma_{\mu \nu}= & \frac{\hbar}{4 \pi N \Omega} \operatorname{Tr}\left\langle\hat{j}_{\mu}\left[G^{+}\left(E_{F}\right)-G^{-}\left(E_{F}\right)\right] \hat{j}_{\nu} G^{-}\left(E_{F}\right)\right. \\
& \left.-\hat{j}_{\mu} G^{+}\left(E_{F}\right) \hat{j}_{\nu}\left[G^{+}\left(E_{F}\right)-G^{-}\left(E_{F}\right)\right]\right\rangle_{\mathrm{c}},
\end{aligned}
$$

where $\Omega$ is the volume of the unit cell, $N$ is the number of sites, $\hat{j}_{\mu}$ is the relativistic current operator, and $G^{ \pm}\left(E_{F}\right)$ are the electronic retarded and advanced Green's functions, respectively, calculated at the Fermi energy $E_{F}$. In Eq. (4) the orbital current term has been omitted as it provides only small corrections to the prevailing contribution arising from the first term in the case of a cubic metallic system [24-26].

The Gilbert damping parameters $\alpha$ are calculated using a Kubo-Greenwood-like equation [27]:

$$
\alpha_{\mu \mu}=-\frac{\hbar \gamma}{\pi M_{s}} \operatorname{Tr}\left\langle\hat{T}_{\mu} \operatorname{Im} G^{+}\left(E_{F}\right) \hat{T}_{\mu} \operatorname{Im} G^{+}\left(E_{F}\right)\right\rangle_{\mathrm{c}},
$$

with the torque operator $\hat{T}_{\mu}$ given by the expression

$$
\hat{T}_{\mu}=\beta\left[\vec{\sigma} \times \hat{e}_{z}\right]_{\mu} B_{x c}(\vec{r}),
$$

with $\hat{e}_{z}$ being the direction of magnetization and $B_{x c}(\vec{r})$ being the spin-dependent part of the potential.

The angular brackets $\langle\cdots\rangle_{c}$ (if applicable) in all expressions above specify the average over temperature-induced spin fluctuations and lattice vibrations treated within the alloy analogy model described in the Appendix A.

\section{RESULTS}

First, we focus on the finite-temperature properties of the electrical resistivity of FeRh. In order to take into account electron-phonon and electron-magnon scattering effects in the calculations, the so-called alloy analogy model $[27,28]$ is used. Within this approach the temperature-induced spin (local moment) and lattice excitations are treated as localized, slowly varying degrees of freedom with temperaturedependent amplitudes. Using the adiabatic approximation in the calculations of transport properties and accounting for the random character of the motions, the evaluation of the thermal average over the spin and lattice excitations in Eq. (4) is reduced to a calculation of the configurational average over the local lattice distortions (averaged within the unit cell) and magnetic moment orientations, $\langle\cdots\rangle_{c}$, using the recently reported approach [27,28], which is based on the coherent potential approximation (CPA) alloy theory [29-31].

To account for the effect of spin fluctuations, which we describe in a way similar to what is done within the disordered local moment (DLM) theory [32], the angular distribution of thermal spin moment fluctuations is calculated using the results of Monte Carlo (MC) simulations. These are based on $a b$ initio exchange coupling parameters and reproduce the finite-temperature magnetic properties for the AFM and FM states in both the low-temperature $\left(T<T_{m}\right)$ and high-temperature ( $T>T_{m}$ ) regions very well [33]. The inset in Fig. 1(a) shows the temperature-dependent magnetization $M(T)$ for one of the two Fe sublattices aligned antiparallel (parallel) to each other in the AFM (FM) state, calculated across the temperature region covering both AFM and FM states of the system. The different behavior of the magnetic order $M(T)$ in the two phases has important consequences for the transport properties, as discussed below.

Figure 1(a) shows the calculated electrical resistivity as a function of temperature $\rho_{x x}(T)$, accounting for the effects of electron scattering from thermal spin and lattice excitations, and compares it with experimental data. There is clearly a rather good theory-experiment agreement, especially concerning the difference $\rho_{x x}^{A F M}\left(T_{m}\right)-\rho_{x x}^{F M}\left(T_{m}\right)$ at the AFM/FM transition, $T_{m}=320 \mathrm{~K}$. The AFM state's resistivity increases more steeply with temperature when compared to that of the FM state, which has also been calculated for temperatures below the metamagnetic transition temperature (dotted line). Note that the experimental measurements have been performed for a sample with $1 \%$ intermixing between the $\mathrm{Rh}$ and $\mathrm{Fe}$ sublattices, leading to a finite residual resistivity at $T \rightarrow 0$ $\mathrm{K}$, and as a consequence, there is a shift of the experimental $\rho_{x x}(T)$ curve with respect to the theoretical one [34]. 


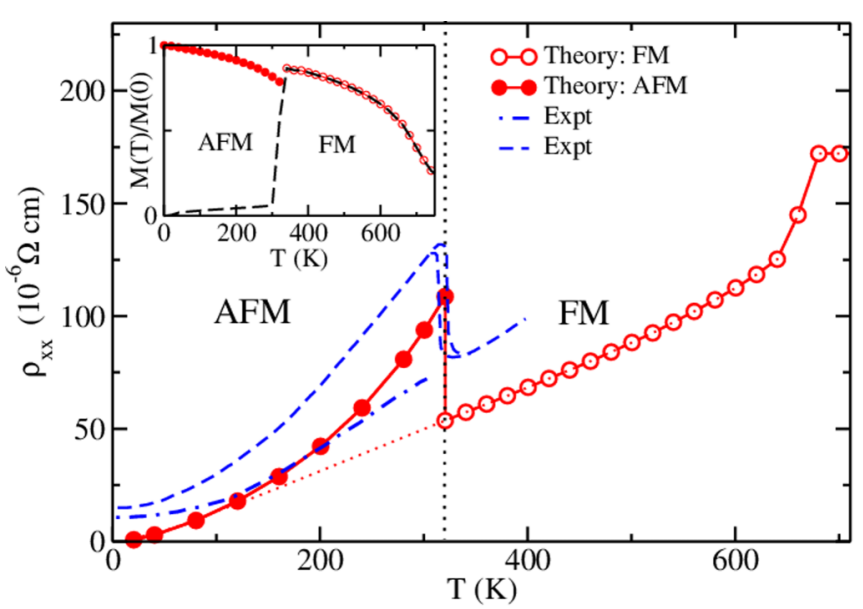

(a)

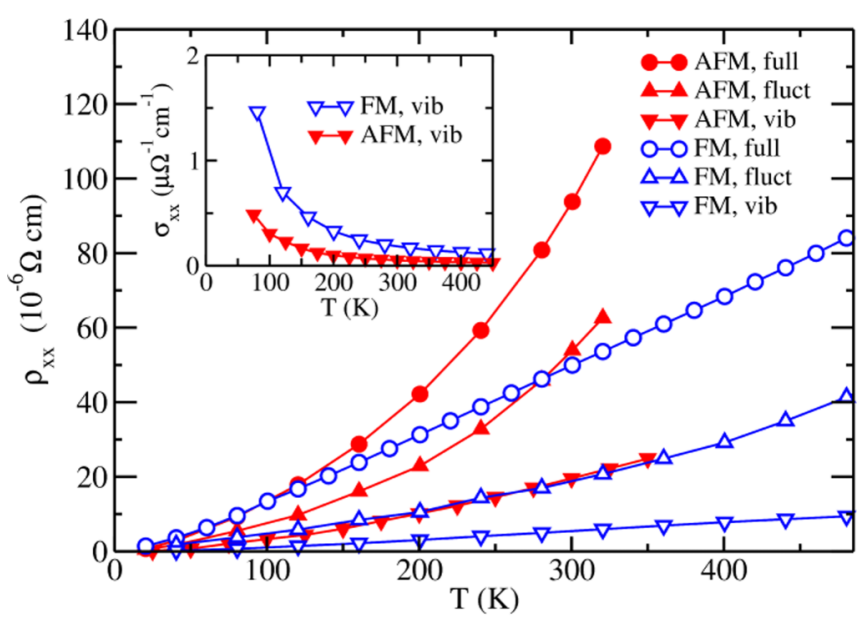

(b)

FIG. 1. (a) Calculated longitudinal resistivity (solid circles: AFM state, open circles: FM state) in comparison with experiment [2]. The dashed line represents the results for $\mathrm{Fe}_{0.49} \mathrm{Rh}_{0.51}$, while the dashdotted line gives results for $(\mathrm{Fe}-\mathrm{Ni})_{0.49} \mathrm{Rh}_{0.51}$ with the $\mathrm{Ni}$ concentration $x=0.05$ to stabilize the FM state at low temperature. The inset represents the relative magnetization of one Fe sublattice as a function of temperature obtained from MC simulations (AFM: solid circles, FM: open circles) and the experimental magnetization curve $M(T)$ (dashed line). (b) Electrical resistivity calculated for the AFM (solid symbols) and FM (open symbols) states accounting for all thermal scattering effects (circles) as well as accounting for effects of lattice vibrations (diamond) and spin fluctuations (squares) separately. The inset shows the temperature-dependent longitudinal conductivity for the AFM and FM states due to only lattice vibrations.

We can separate out the contributions of spin fluctuations and lattice vibrations to the electrical resistivities, $\rho_{x x}^{\text {fluc }}(T)$ and $\rho_{x x}^{\mathrm{vib}}(T)$, respectively. These two components have been calculated for finite temperatures while keeping the atomic positions undistorted to find $\rho_{x x}^{\text {fluc }}(T)$ and with fixed collinear orientations of all magnetic moments to find $\rho_{x x}^{\mathrm{vib}}(T)$, respectively. The results for the AFM and FM states are shown in Fig. 1(b), where again the FM (AFM) state has also been considered below (above) the transition temperature $T_{m}$. For both magnetic states the local moment fluctuations have a dominant impact on the resistivity. One can also see that both components, $\rho_{x x}^{\text {fluc }}(T)$ and $\rho_{x x}^{\mathrm{vib}}(T)$, in the AFM state have a steeper increase with temperature than those of the FM state.

The origin of this behavior can be clarified by referring to Mott's model [35] with its distinction between delocalized $s p$ electrons, which primarily determine the transport properties owing to their high mobility, and the more localized $d$ electrons. Accordingly, the conductivity should depend essentially on (see, e.g., [36]) (i) the carrier (essentially, $s p$ character) concentration $n$ and (ii) the relaxation time $\tau \sim$ $\left[V_{\text {scatt }}^{2} n\left(E_{F}\right)\right]^{-1}$, where $V_{\text {scatt }}$ is the average scattering potential and $n\left(E_{F}\right)$ is the total density of states at the Fermi level. This model has been used, in particular, for qualitative discussions of the origin of the giant magnetoresistance (GMR) effect in heterostructures consisting of magnetic layers separated by nonmagnetic spacers. In this case the GMR effect can be attributed to the spin-dependent scattering of conduction electrons, which leads to a dependence of the resistivities on the relative orientation of magnetic layers, parallel or antiparallel, assuming the electronic structure of nonmagnetic spacer remains unchanged. These arguments, however, cannot be straightforwardly applied to $\mathrm{CsCl}$-structured $\mathrm{FeRh}$, even though it can be pictured as a layered system with oneatom-thick layers, since the electronic structure of $\mathrm{FeRh}$ shows strong modifications across the AFM-FM transition as discussed, for example, by Kobayashi et al. [11] to explain the large MR effect in FeRh.

We use the calculated density of states at the Fermi level as a measure of the concentration of the conducting electrons. The change in the carrier concentration at the AFM-FM transition can therefore be seen from the modification of the $s p$ DOS at the Fermi level. The element-projected spin-resolved $s p$ DOS $n_{s p}(E)$, calculated for both FM and AFM states at different temperatures, is shown in Fig. 2. At low temperature, for

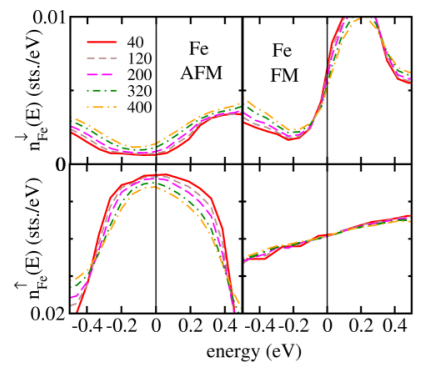

(a)

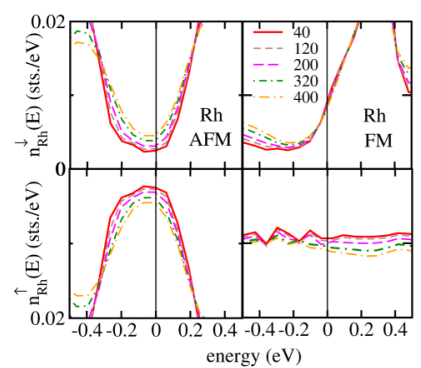

(c)

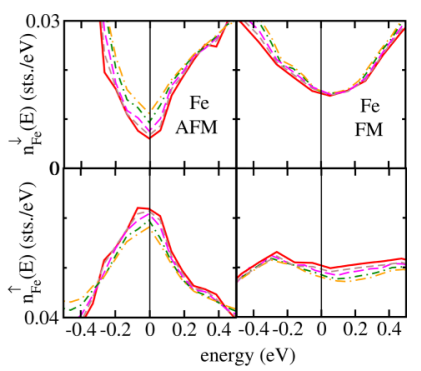

(b)

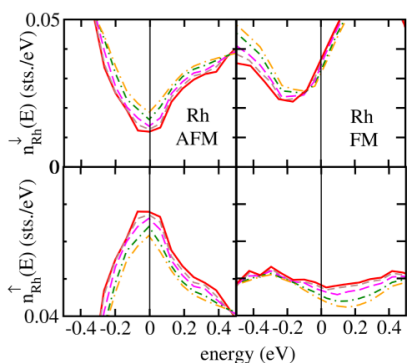

(d)
FIG. 2. Comparison of the temperature-dependent densities of states (DOSs) for the FM and AFM states of FeRh for $T=40-400 \mathrm{~K}$ : (a) Fe $s$ DOS, (b) Fe $p$ DOS, (c) Rh $s$ DOS, and (d) Rh $p$ DOS. 
both $\mathrm{Fe}$ and $\mathrm{Rh}$ sublattices, the $s p$ DOS at $E_{F}$ is higher in the FM state than in the AFM state, $n_{s p}^{F M}\left(E_{F}\right)>n_{s p}^{A F M}\left(E_{F}\right)$. This gives the first hint concerning the origin of the large difference between the FM and AFM conductivities in the low-temperature limit [see inset for $\sigma_{x x}^{\mathrm{vib}}$ in Fig. 1(b)]. In this case the relaxation time $\tau$ is still long owing to the low level of both lattice vibrations and spin fluctuations, which determines the scattering potential $V_{\text {scatt }}$. For both magnetic states the decrease in the conductivity with rising temperature is caused by the increase of scattering processes and consequent decrease of the relaxation time. At the same time, the conductivity difference, $\Delta \sigma(T)=\sigma_{x x}^{\mathrm{vib} F M}(T)-$ $\sigma_{x x}^{\mathrm{vib}, A F M}(T)$, reduces with an increase in temperature. This effect can partially be attributed to the temperature-dependent changes in the electronic structure (disorder smearing of the electronic states) reflected by changes in the density of states at the Fermi level [34] (see Fig. 2). Despite this, up to the transition temperature, $T=T_{m}$, the difference $\Delta \sigma(T)$ is rather pronounced, leading to a significant change in the resistivity at $T=T_{m}$.

One has to stress that in calculating the contribution of spin moment fluctuations to the resistivity, the different temperature-dependent behaviors of the magnetic order in the FM and AFM states must be taken into account. This means that at the critical point, $T=T_{m}$, the smaller sublattice magnetization in the AFM state describes a more pronounced magnetic disorder when compared to the FM state, which leads to both a smaller relaxation time and shorter mean free path. The result is a higher resistivity in the AFM state.

The different mean-free-path lengths in the FM and AFM states at a given temperature can be analyzed using the BSF $A_{B}(\vec{k}, E)$, calculated for $E=E_{F}$, since the electronic states at the Fermi level give the contribution to the electrical conductivity. For a system with thermally induced spin fluctuations and lattice displacements the BSF has features with finite width from which the mean-free-path length of the electrons can be inferred. Figure 3 shows an intensity contour plot for the BSF of FeRh averaged over local-moment configurations appropriate for the FM and AFM states just above and just below the FM-AFM transition, respectively. Figure 3(a) shows the AFM Bloch spectral function, whereas Figs. 3(b) and 3(c) show the sharper features of the spin-polarized BSF of the FM state, especially for the minority-spin states. This implies a longer electronic mean free path in the FM state in comparison to that in the AFM state, which is consistent with the drop in resistivity.

Finally, we discuss the behavior of the electrical resistivity of FM-ordered FeRh in the vicinity to the Curie temperature. First of all, once the temperature has been raised above the Curie temperature and the system is in a magnetically disordered state, there is no longer a contribution from the spin fluctuations to the increase in the resistivity $\rho(T)$ when $T$ is increased further. The transition to the PM state results therefore in an abrupt decrease of the rate of increase of $\rho(T)$ with temperature (see Fig. 1). This effect, observed also in $\mathrm{Fe}$ and $\mathrm{Ni}$, has been discussed previously [28]. Below $T_{C}$, the sharp increase of the resistivity as the Curie temperature is approached is a consequence of the fast increase in the amplitude of transverse spin fluctuations in this temperature region. Figure 4 demonstrates the impact of thermally induced

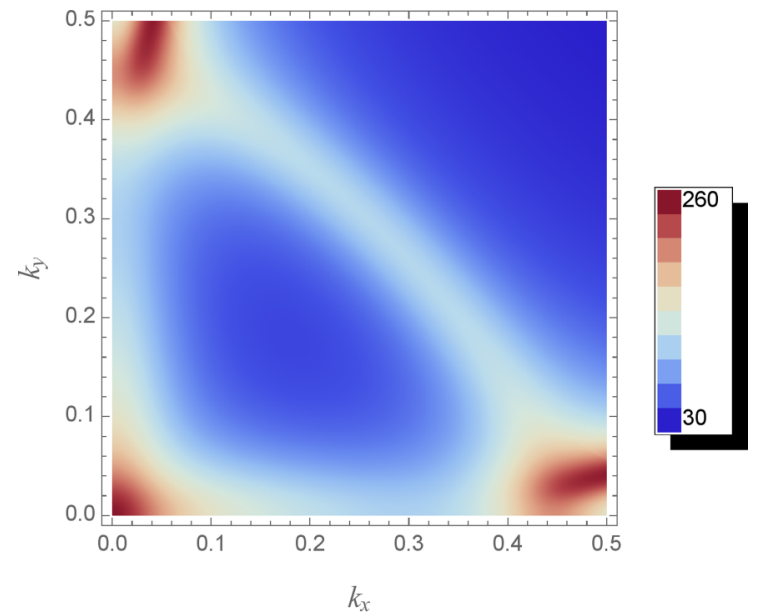

(a)

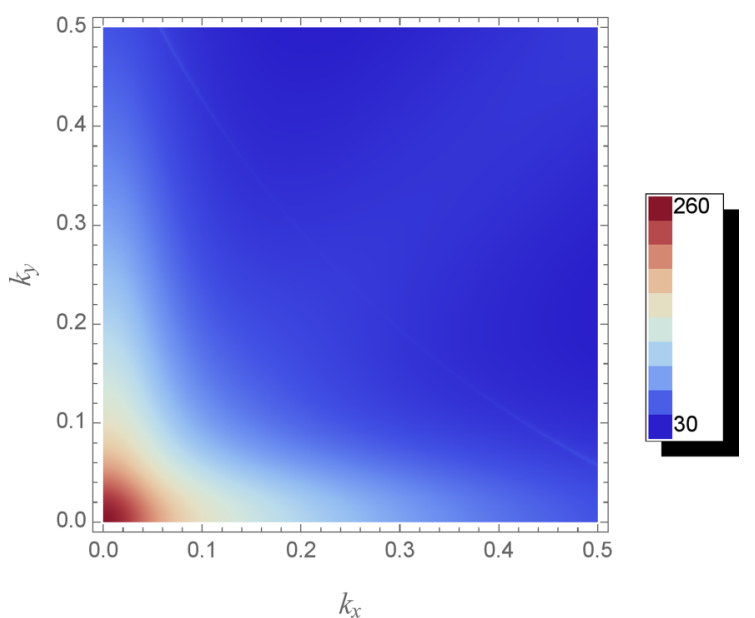

(b)

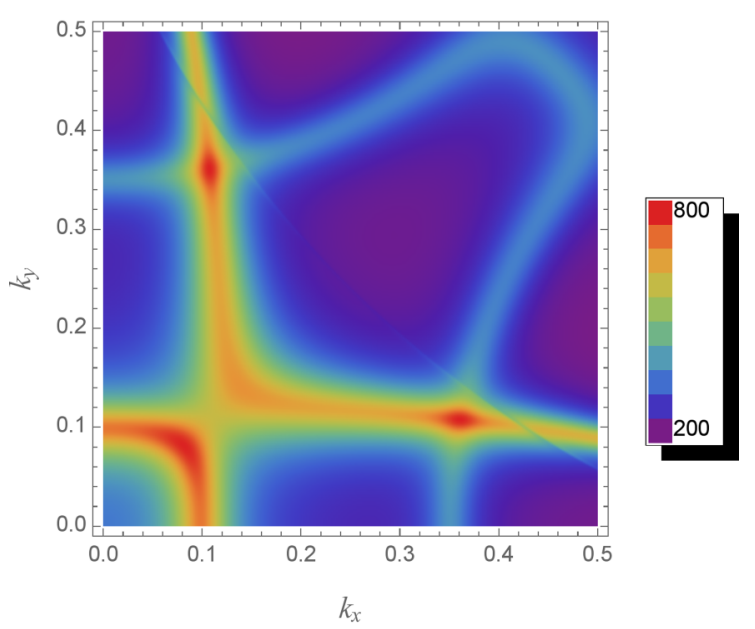

(c)

FIG. 3. Bloch spectral function (in units of states/Ry) of FeRh calculated (a) for the AFM state at $T=300 \mathrm{~K}$ and for the FM state resolved into (b) majority-spin and (c) minority-spin electron components, calculated for $T=320 \mathrm{~K}$. The finite width of this feature determines the electronic mean free paths.

magnetic disorder on the electronic structure, leading to an increase in smearing of the electron energy bands when the temperature changes from 600 to $700 \mathrm{~K}$. As discussed above, 

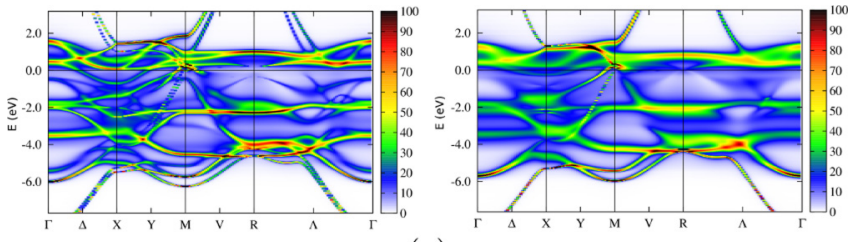

(a)
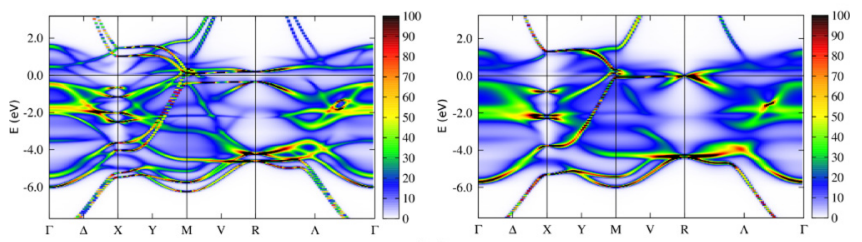

(b)
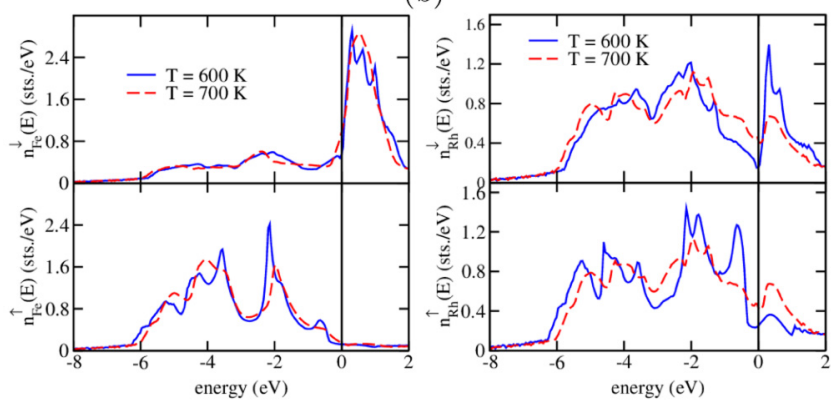

(c)

FIG. 4. Element-resolved BSF on (a) Fe and (b) Rh sites in FeRh calculated for the FM (left) and PM (right) states at finite temperatures $T=600 \mathrm{~K}$ and $T=700 \mathrm{~K}$, respectively; (c) comparison of the element-resolved Fe (left) and Rh (right) DOS calculated for the FM (solid line) and PM (dashed line) states at finite temperatures $T=600 \mathrm{~K}\left(M / M_{0}=0.66\right)$ and $T=700 \mathrm{~K}\left(M / M_{0}=0\right)$.

this observation is connected to the shortened lifetime of the electronic states that causes an increase in the electrical resistivity. Clearly, the differences in the $\rho(T)$ behavior in the vicinity of $T_{C}$ for different systems stem from specific features of their electronic structures relevant to their PM states. For example, there is (i) magnetic "local-moment" disorder in the case of pure $\mathrm{Fe}$, (ii) a Pauli paramagnetic state in the case of pure $\mathrm{Ni}$, and (iii) magnetic local-moment disorder on the $\mathrm{Fe}$ sublattice and disappearance of spin polarization on the $\mathrm{Rh}$ sublattice in the case of FeRh. Figure 4(b) demonstrates the induced spin splitting of the $\mathrm{Rh}$ electronic states in FeRh, in particular around the Fermi level, at $T<T_{C}(T=600 \mathrm{~K}$, left panel). This splitting disappears above $T_{C}(T=700 \mathrm{~K}$, right panel), so that the Rh DOS increases at the Fermi level [Fig. 4(c), right panel]. This leads in turn to the sharp increase in the resistivity as the critical temperature is approached since $\rho(T)$ is inversely proportional to the relaxation time $\tau$, i.e., $\rho(T) \sim\left[V_{\text {scatt }}^{2} n\left(E_{F}\right)\right]$ (see discussions above). It is also worth mentioning the combined effect of both scattering channels that arise from spin fluctuations and lattice vibrations. The latter contribution is rather small (see Fig. 1), and consequently, $\rho(T)$ has a temperature dependence determined essentially by the spin fluctuations. In the case of Fe [28], on the other hand, both contributions are comparable, and lattice vibrations lead to a rather pronounced smearing of the electronic states at $E_{F}$ when the temperature approaches $T_{C}$, which conceals the impact of the electron scattering from the

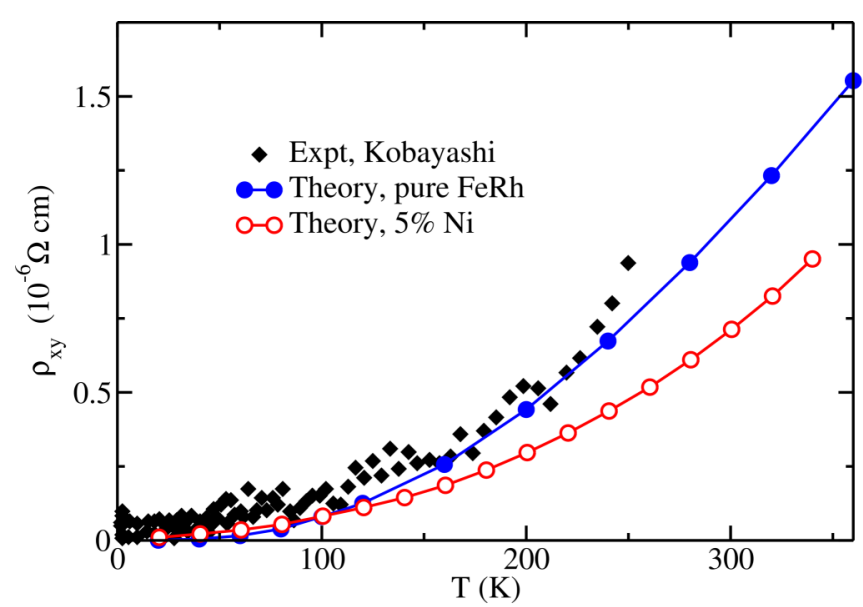

(a)

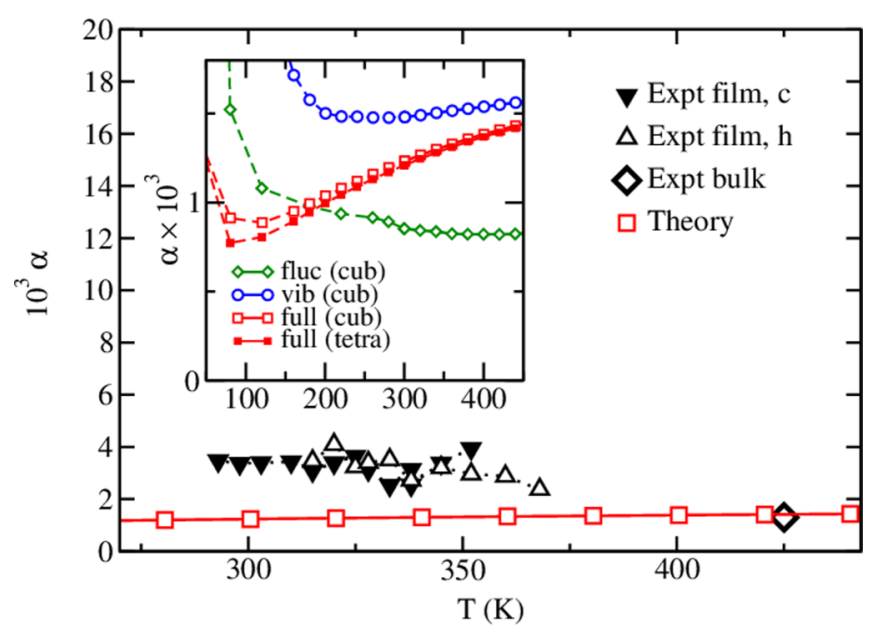

(b)

FIG. 5. (a) The temperature dependence of the anomalous Hall resistivity for the FM state of $\left(\mathrm{Fe}_{0.95} \mathrm{Ni}_{0.05}\right) \mathrm{Rh}$ in comparison with experimental data [11]; (b) Gilbert damping parameter as a function of temperature: theory accounting for all thermal contributions (squares) in comparison with the experimental results for a thick-film system (50 nm; open diamonds) [37] and for an FeRh thin film deposited on a $\mathrm{MgO}(001)$ surface (upward and downward triangles). Upward and downward triangles represent data for heating (h) and cooling (c) cycles, respectively (for details see Appendix B). The inset represents the results for the individual sources for the Gilbert damping, i.e., lattice vibrations (circles) and spin fluctuations (diamonds). The total $\alpha$ values calculated for the FeRh crystal without (cub) and with tetragonal (tetra) distortions $(c / a=1.016)$ are shown by open and solid squares, respectively. Gilbert damping for the FM phase is shown by dashed lines in the temperature region below the metamagnetic transition temperature.

spin fluctuations. As a result, the total $\rho(T)$ has an almost linear increase up to $T_{C}$.

In particular concerning technical applications of FeRh, it is interesting to study further temperature-dependent response properties. In Fig. 5(a) we show our calculations of the total anomalous Hall resistivity for FeRh in the FM state, represented by the off-diagonal term $\rho_{x y}$ of the resistivity tensor, and compare it with experimental data [11]. As the 
FM state is unstable in pure FeRh at low temperatures, the measurements were performed for $\left(\mathrm{Fe}_{0.965} \mathrm{Ni}_{0.035}\right) \mathrm{Rh}$, for which the FM state has been stabilized by Ni doping. The calculations have been performed both for the pure FeRh compound and for FeRh with $5 \% \mathrm{Ni}$ doping, $\left(\mathrm{Fe}_{0.95} \mathrm{Ni}_{0.05}\right) \mathrm{Rh}$, which theory finds to be ferromagnetically ordered down to $T=0 \mathrm{~K}$. As can be seen, the magnitude of $\rho_{x y}(T)$ increases in a more pronounced way for the undoped system. Nevertheless, both results are in rather good agreement with experiment.

In addition to temperature-dependent transport properties linear response calculations with the inclusion of relativistic effects enable us to present results for Gilbert damping, which plays a crucial role for spin dynamics. The experimental data shown in Fig. 5(b) by triangular symbols represent results for rather thin FeRh films $(d=10 \mathrm{~nm})$ deposited on top of a $\mathrm{MgO}(001)$ substrate (see experimental details described in Appendix B). Upward and downward triangles in Fig. 5(b) represent the Gilbert damping obtained for heating and cooling cycles, respectively. The FeRh unit cell with a lattice constant $\sqrt{2}$ times smaller than that of $\mathrm{MgO}$ is rotated around the $z$ axis by $45^{\circ}$ with respect to the $\mathrm{MgO}$ cell. Because of this, a compressive strain occurs in the FeRh film. From the experimental data [38], this implies a tetragonal distortion of the FM FeRh unit cell with $c / a=1.016$.

The $\alpha$ calculations have been performed for the FM state taking into account all temperature-induced effects, i.e., spin fluctuations and lattice vibrations [28,39]. As one can see in Fig. 5(b), these results are in good agreement with the experimental value (shown by a diamond) for a thick (bulk) film where $\alpha$ was measured as 0.0012 at $T=420 \mathrm{~K}$ [37]. However, the calculated $\alpha$ values are smaller by a factor of 3 when compared to the experimental data measured for the thinner $10-\mathrm{nm}$ film. Accounting for the tetragonal distortion results in a rather weak change for the calculated $\alpha$, as can be seen in the inset of Fig. 5(b) (solid squares). Therefore, the discrepancies between theory and experiment have to be attributed partially to surface and finite-size effects, as discussed, for example, by Barati et al. [40], which are not accounted for within the present calculations. Another reason for the discrepancies can be associated with the inhomogeneities presented in the sample. Note also that the measurements represented in Fig. 5(b) have been performed in the vicinity of the metamagnetic AFM-FM transition. In this temperature region the FM state is not uniform, as discussed, for example, by Baldasseroni et al. [41], who observed the mixed-phase (FM+AFM) state close to the $T_{m}$ transition temperature. Evidently, this can also lead to an increase in the Gilbert damping in this temperature region when compared to the pure FM state considered in the calculations.

The separate contributions to the Gilbert damping due to spin fluctuations and lattice vibrations are shown in the inset of Fig. 5(b) for a range of temperatures extended to low temperatures beyond those measured by experiment. As discussed in the literature, magnetization dissipation at low temperature is well described via the breathing Fermi-surface model for pure elemental materials and ordered compounds [28,39,42]. In this regime the temperature dependence of the Gilbert damping is directly connected to the relaxation time parameter of the electronic subsystem, which in turn is determined by the dominating spin-conserving electron scattering that arises from lattice vibrations, $V_{\text {vib }}^{2}$, and spin fluctuations, $V_{\text {flu }}^{2}$. In this low-temperature regime (as discussed in Appendix $\mathrm{C}$ ), $\alpha \sim\left[V_{\mathrm{vib}}^{2}+V_{\mathrm{flu}}^{2}\right]^{-1}$. The thermally induced increase in the amplitude of lattice vibrations and spin fluctuations results in an increase in the effective scattering cross section for the electrons and hence a decrease in the Gilbert damping. Based on the expressions given in Appendix $\mathrm{C}$, one can consider individual contributions from different scattering channels at low temperature. Thus, since $\alpha \sim\left(\alpha_{\text {vib }}^{-1}+\alpha_{\text {fluc }}^{-1}\right)^{-1}$, one can say that the higher rate of decrease with rising temperature for $\alpha$ is associated with the scattering mechanism which has the larger scattering cross section. In particular, at $T \approx 200 \mathrm{~K}$, the Gilbert damping associated with spin fluctuations is appreciably smaller than that due to lattice vibrations [see inset in Fig. 5(b)]. This implies a large decrease at $T<200 \mathrm{~K}$ of $\alpha(T)$ with an increase in $T$, as seen in the inset in Fig. 5(b). This clearly shows (see Appendix C) the dominant role of spin fluctuations for the Gilbert damping in the low-temperature regime, leading to a similar behavior for the total Gilbert damping [squares in Fig. 5(b)]. Moreover, it can be seen that the total $\alpha$ accounting for both scattering channels is still smaller in the low-temperature regime owing to the increased effective scattering cross section.

The "resistivitylike" behavior at higher temperatures, i.e., $\alpha$ growing with rising temperature, reflects the increasing role of the interband transitions which determines a dominating spin-flip dissipation mechanism [43]. In this regime, as seen in Fig. 5(b), the increase in the total Gilbert damping with rising temperature is predominantly determined by electron scattering from lattice vibrations, demonstrating the leading role of this scattering channel for the Gilbert damping at high temperatures. Note that the spin fluctuations in the temperature region shown in Fig. 5(b) lead to a weak decrease of $\alpha(T)$ with an increasing temperature, indicating a small contribution to the spin-flip dissipation mechanism.

\section{SUMMARY}

In summary, we have presented ab initio calculations for the finite-temperature transport properties of the FeRh compound. A steep increase in the electric resistivity has been obtained for the AFM state, leading to a pronounced drop in resistivity at the AFM to FM transition temperature. This effect can be attributed partially to the difference in the electronic structure of FeRh in the FM and AFM states, as well as to a faster increase in the amplitude of spin fluctuations caused by temperature in the AFM state. Further calculated temperaturedependent response properties such as the anomalous Hall effect (AHE) resistivity and the Gilbert damping parameter for the FM system also show good agreement with experimental data. This gives additional confidence in the model used to account for thermal lattice vibrations and spin fluctuations.

\section{ACKNOWLEDGMENTS}

Financial support from the DFG via SFB 689 (Spinphänomene in reduzierten Dimensionen) and from the EPSRC (UK; Grant No. EP/J006750/1) is gratefully acknowledged. 


\section{APPENDIX A: TREATMENT OF THERMAL LATTICE DISPLACEMENT AND SPIN FLUCTUATIONS}

To account for the impact of the thermal lattice vibrations and spin fluctuations, the alloy analogy model is used in the present work. The multiple-scattering theory allows us to describe the uncorrelated local thermal atomic displacements and spin moment deviations from their equilibrium, within the single-site CPA alloy theory. This implies the reduction of the calculation of the thermal average to the calculation of a configurational average in full analogy with random alloy systems [28]. Within this approach the coherent scattering path operator is defined as

$$
\underline{\tau}_{\mathrm{CPA}}=\sum_{v=1}^{N_{v f}} x_{v} x_{f} \underline{\tau}_{v f},
$$

with summation over all types of local lattice vibrations and spin fluctuations with the corresponding probabilities $x_{v}$ and $x_{f}$ [28]. The underline indicates matrices with respect to the combined index $\Lambda$. The $\underline{\tau}_{v f}$ operators are defined through the corresponding single-site scattering matrices $t_{v f}^{\text {loc }}[28]$ :

$$
\underline{t}_{v f}=\underline{U}\left(\Delta \vec{R}_{v}\right) \underline{R}\left(\hat{e}_{f}\right) \underline{t} \underline{R}\left(\hat{e}_{f}\right)^{-1} \underline{U}\left(\Delta \vec{R}_{v}\right)^{-1} .
$$

Here $\underline{R}(\hat{e})$ is a rotation matrix for the transformation from the local to the global frame of reference. The so-called $U$ transformation matrix $\underline{U}\left(\Delta \vec{R}_{v}^{q}\right)$ for each atomic $q$ site in the unit cell is given by $[4 \overline{4,45]}$

$$
U_{L L^{\prime}}\left(\Delta \vec{R}_{v}^{q}\right)=4 \pi \sum_{L^{\prime \prime}} i^{l+l^{\prime \prime}-l^{\prime}} C_{L L^{\prime} L^{\prime \prime}} j_{l^{\prime \prime}}\left(\left|\Delta \vec{R}_{v}^{q}\right| k\right) Y_{L^{\prime \prime}}(\hat{s}),
$$

where $L=(l, m)$ represents the nonrelativistic angular momentum quantum numbers, $j_{l}(x)$ is a spherical Bessel function, $Y_{L}(\hat{r})$ is real spherical harmonics, $C_{L L^{\prime} L^{\prime \prime}}$ is the corresponding Gaunt number, and $k=\sqrt{E}$ is the electronic wave vector. The amplitude of atomic displacements $\left|\Delta \vec{R}_{v}^{q}\right|$ is represented by the temperature-dependent rms displacement $\left(\left\langle u^{2}\right\rangle_{T}\right)^{1 / 2}$ according to

$$
\sum_{v=1}^{N_{v}} x_{v}^{q}\left|\Delta \vec{R}_{v}^{q}(T)\right|^{2}=\left\langle u_{q}^{2}\right\rangle_{T} .
$$

Basically, the mean-square displacement of the atom $q$ along the direction $\mu(\mu=x, y, z)$ can be evaluated within phonon calculations [46]. However, in the present work we have used the approach based on Debye's theory with the Debye temperature $\Theta_{D}$ taken from experiment [47]. In this case the individual mean-square displacements for different atomic types in the unit cell are not well defined. Moreover, their relative magnitudes can change as a function of temperature as a consequence of different ratios of the amplitude of displacements for different types of atoms, associated with acoustic and optical phonon modes in the limit of small wave vector $\vec{q}$, as well as with the phonon modes with $\vec{q}$ approaching the boundary of the Brillouin zone $\vec{G} / 2$ (see, e.g., Ref. [46]). Because of the lack of such information, we have used an approximation based on the averaged mean-square displacement. This implies that the mean-square displacements for both types, $\mathrm{Fe}$ and $\mathrm{Rh}$, are equal and are given by the expression $[48,49]$

$$
\left\langle u_{\mu}^{2}\right\rangle_{T}=\frac{1}{4} \frac{3 h^{2}}{\pi^{2} M k_{\mathrm{B}} \Theta_{D}}\left[\frac{\Phi\left(\Theta_{D} / T\right)}{\Theta_{D} / T}+\frac{1}{4}\right],
$$

with $\Phi\left(\Theta_{D} / T\right)$ being the Debye function. In spite of the simplicity, this approach gives results in rather good agreement with experimental data for disordered alloys as well as for ordered compounds, as was shown previously [39,50]. As a consequence of the above-mentioned temperature-dependent properties of the mean-square displacements, the difference between the experimental and theoretical resistivities for the ordered FeRh compound [see Fig. 1(a) in the main text] can be partially attributed to the present simplification used for the evaluation of mean-square displacements.

\section{APPENDIX B: EXPERIMENTAL DETAILS OF GILBERT DAMPING}

FeRh films were grown on (001)-oriented single-crystal $\mathrm{MgO}$ substrates using dc magnetron sputtering. The base pressure of the chamber was $2 \times 10^{-5} \mathrm{~Pa}$. The substrates were kept at $573 \mathrm{~K}$ for $30 \mathrm{~min}$. Then 10-nm FeRh were deposited with a growth pressure of $0.7 \mathrm{~Pa}$ Ar corresponding to stoichiometric $\mathrm{Fe}_{51} \mathrm{Rh}_{49}$ films [51]. The sputtering power is $30 \mathrm{~W}$ for 3-inch $\mathrm{Fe}_{50} \mathrm{Rh}_{50}$ targets. Afterwards, the films were heated to $1023 \mathrm{~K}$ and annealed for $100 \mathrm{~min}$. When the films were cooled down to room temperature, they were capped with 5-nm Al in situ.

The experimental data were obtained by field-swept ferromagnetic resonance measurements of a 25-nm FeRh film grown on $\mathrm{MgO}(001)$ and capped by $5-\mathrm{nm} \mathrm{Al}$ in the out-ofplane configuration for frequencies from 5 to $24 \mathrm{GHz}$. The temperature was controlled by heating through the substrate, and the measured absorption spectra were fitted to a Lorentzian line shape [52] in order to obtain the linewidth $\Delta H$. The damping parameter $\alpha$ was determined from the frequency dependence of $\Delta H$, as demonstrated by Mancini et al. [37] and Heinrich et al. [53].

\section{APPENDIX C}

To discuss the temperature-dependent behavior of Gilbert damping in more detail one can represent the expression in Eq. (5) in terms of the Bloch spectral function $A(E, \vec{k}, n)$, following the corresponding discussions by Kamberský [54] and Gilmore et al. [55]. According to these authors, the leading contribution to the Gilbert damping in the lowtemperature limit is associated with the intraband scattering given by $[54,55]$

$$
\begin{aligned}
\alpha_{\text {intra }} \sim & \sum_{n} \int \frac{d^{3} k}{(2 \pi)^{3}}\left|\Gamma_{n n}^{-}(\vec{k})\right|^{2} \\
& \times \int d E A(E, \vec{k}, n) A(E, \vec{k}, n)\left(-\frac{d f(E)}{d E}\right),
\end{aligned}
$$

with

$$
A(E, \vec{k}, n)=\frac{w_{\vec{k}, n}}{\left(E-E_{\vec{k}, n}\right)^{2}+w_{\vec{k}, n}^{2}},
$$


where $\Gamma_{n n}^{-}$is the matrix element of the transverse torque operator and $w_{\vec{k}, n}^{2}$ is related to the imaginary part of the the scattering self-energy [54]. In the present work we discuss two contributions due to various electron scattering channels, i.e., due to lattice vibrations with $\operatorname{Im} \Sigma_{\vec{k}, n}^{\mathrm{vib}} \sim\left(\tau_{\vec{k}, n}^{\mathrm{vib}}\right)^{-1}$ and due to spin fluctuations with $\operatorname{Im} \Sigma_{\vec{k}, n}^{\mathrm{flu}} \sim\left(\tau_{\vec{k}, n}^{\mathrm{flu}}\right)^{-1}$, and the relaxation times, $\tau_{\vec{k}, n}^{\mathrm{vib}}$ and $\tau_{\vec{k}, n}^{\mathrm{flu}}$, corresponding to the different scattering channels. With this, $w_{\vec{k}, n}^{2}$ can be represented by the effective relaxation time $\left(\tau^{\text {eff }}\right)^{-1}=\left(\tau^{\mathrm{vib}}\right)^{-1}+\left(\tau^{\mathrm{flu}}\right)^{-1}$. As was shown in Refs. [54,55], after integration over the energies, Eq. (C1) can be reduced to

$$
\alpha_{\text {intra }} \sim \tau_{\text {eff }} \sum_{n} \int \frac{d^{3} k}{(2 \pi)^{3}}\left|\Gamma_{n n}^{-}(\vec{k})\right|^{2} .
$$

According to the discussions above, we have $\tau^{\text {flu }} \sim$ $\left[V_{\mathrm{flu}}^{2} n\left(E_{F}\right)\right]^{-1}$ and $\tau^{\mathrm{vib}} \sim\left[V_{\mathrm{vib}}^{2} n\left(E_{F}\right)\right]^{-1}$, leading to the following dependence: $\alpha \sim \tau_{\text {eff }} \sim\left[V_{\text {vib }}^{2}+V_{\text {flu }}^{2}\right]^{-1}$. The expression in Eq. (C2) can also be reduced to the form used for discussions of the Gilbert damping within the breathing Fermi-surface model [55-57] that describes well the temperature-dependent behavior $\alpha(T)$ in the low-temperature regime.
The interband contribution in terms of Bloch spectral function is given by the expression $[54,55]$

$$
\begin{aligned}
\alpha_{\text {inter }} \sim & \sum_{n \neq m} \int \frac{d^{3} k}{(2 \pi)^{3}}\left|\Gamma_{n m}^{-}(\vec{k})\right|^{2} \\
& \times \int d E A(E, \vec{k}, n) A(E, \vec{k}, m)\left(-\frac{d f(E)}{d E}\right) .
\end{aligned}
$$

At low temperature this contribution increases with temperature as $\alpha_{\text {inter }} \sim \tau_{\text {eff }}^{-1} \sim\left[V_{\text {vib }}^{2}+V_{\text {flu }}^{2}\right]$ [54,55] and above a certain temperature $T_{m}$ becomes the dominating part of the Gilbert damping. This leads to the minimum for $\alpha(T)$ at $T_{m}$, which is determined by both $V_{\text {vib }}$ and $V_{\text {flu }}$ scattering amplitudes in the case of the total $\alpha$ and by only $V_{\text {vib }}$ or $V_{\text {flu }}$ scattering amplitudes in the case of individual contributions due to lattice vibrations and spin fluctuations, respectively, resulting in different positions of the minima in these three cases. Finally, it should be noted that the contributions to $\alpha_{\text {inter }}$ due to lattice vibrations and spin fluctuations are additive, in contrast to $\alpha_{\text {intra }}$. In this case, one gets an increase with temperature of the total $\alpha(T)$ larger than in the case of separate contributions due to different scattering channels.
[1] J. S. Kouvel and C. C. Hartelius, J. Appl. Phys. 33, 1343 (1962).

[2] N. Baranov and E. Barabanova, J. Alloys Compd. 219, 139 (1995).

[3] P. A. Algarabel, M. R. Ibarra, C. Marquina, A. del Moral, J. Galibert, M. Iqbal, and S. Askenazy, Appl. Phys. Lett. 66, 3061 (1995).

[4] M. A. de Vries, M. Loving, A. P. Mihai, L. H. Lewis, D. Heiman, and C. H. Marrows, New J. Phys. 15, 013008 (2013).

[5] P. H. L. Walter, J. Appl. Phys. 35, 938 (1964).

[6] J. S. Kouvel, J. Appl. Phys. 37, 1257 (1966).

[7] R. Barua, F. Jimnez-Villacorta, and L. H. Lewis, Appl. Phys. Lett. 103, 102407 (2013).

[8] W. Lu, N. T. Nam, and T. Suzuki, J. Appl. Phys. 105, 07 A904 (2009).

[9] I. Suzuki, T. Naito, M. Itoh, T. Sato, and T. Taniyama, J. Appl. Phys. 109, 07C717 (2011).

[10] M. Knülle, D. Ahlers, and G. Schütz, Solid State Commun. 94, 267 (1995).

[11] Y. Kobayashi, K. Muta, and K. Asai, J. Phys.: Condens. Matter 13, 3335 (2001).

[12] J. Kudrnovský, V. Drchal, and I. Turek, Phys. Rev. B 91, 014435 (2015).

[13] H. Ebert et al. The Munich SPR-KKR package, version 6.3, http://olymp.cup.uni-muenchen.de/ak/ebert/SPRKKR.

[14] H. Ebert, D. Ködderitzsch, and J. Minár, Rep. Prog. Phys. 74, 096501 (2011).

[15] J. P. Perdew, K. Burke, and M. Ernzerhof, Phys. Rev. Lett. 77, 3865 (1996).

[16] J. S. Faulkner and G. M. Stocks, Phys. Rev. B 21, 3222 (1980).

[17] P. Weinberger, Electron Scattering Theory for Ordered and Disordered Matter (Oxford University Press, Oxford, 1990).

[18] H. Ebert, in Electronic Structure and Physical Properties of Solids, edited by H. Dreyssé, Lecture Notes in Physics Vol. 535 (Springer, Berlin, 2000), p. 191.
[19] M. E. Rose, Relativistic Electron Theory (Wiley, New York, 1961).

[20] P. Strange, J. Staunton, and B. L. Gyorffy, J. Phys. C 17, 3355 (1984).

[21] R. Feder, F. Rosicky, and B. Ackermann, Z. Phys. B 52, 31 (1983).

[22] P. Středa, J. Phys. C 15, L717 (1982).

[23] S. Lowitzer, D. Ködderitzsch, and H. Ebert, Phys. Rev. B 82, 140402(R) (2010).

[24] T. Naito, D. S. Hirashima, and H. Kontani, Phys. Rev. B 81 , 195111 (2010).

[25] S. Lowitzer, M. Gradhand, D. Ködderitzsch, D. V. Fedorov, I. Mertig, and H. Ebert, Phys. Rev. Lett. 106, 056601 (2011).

[26] I. Turek, J. Kudrnovský, and V. Drchal, Phys. Rev. B 86, 014405 (2012).

[27] H. Ebert, S. Mankovsky, D. Ködderitzsch, and P. J. Kelly, Phys. Rev. Lett. 107, 066603 (2011).

[28] H. Ebert, S. Mankovsky, K. Chadova, S. Polesya, J. Minár, and D. Ködderitzsch, Phys. Rev. B 91, 165132 (2015).

[29] B. Velický, Phys. Rev. 184, 614 (1969).

[30] W. H. Butler, Phys. Rev. B 31, 3260 (1985).

[31] I. Turek, J. Kudrnovský, V. Drchal, L. Szunyogh, and P. Weinberger, Phys. Rev. B 65, 125101 (2002).

[32] B. L. Gyorffy, A. J. Pindor, J. Staunton, G. M. Stocks, and H. Winter, J. Phys. F 15, 1337 (1985).

[33] S. Polesya, S. Mankovsky, D. Ködderitzsch, J. Minár, and H. Ebert, Phys. Rev. B 93, 024423 (2016).

[34] J. B. Staunton, R. Banerjee, M.dos Santos Dias, A. Deak, and L. Szunyogh, Phys. Rev. B 89, 054427 (2014).

[35] N. F. Mott, Adv. Phys. 13, 325 (1964).

[36] E. Y. Tsymbal, D. G. Pettifor, and S. Maekawa, in Handbook of Spin Transport and Magnetism, edited by E. Y. Tsymbal and I. Zutić (Taylor and Francis, New York, 2012), p. 95. 
[37] E. Mancini, F. Pressacco, M. Haertinger, E. E. Fullerton, T. Suzuki, G. Woltersdorf, and C. H. Back, J. Phys. D 46, 245302 (2013).

[38] C. Bordel, J. Juraszek, D. W. Cooke, C. Baldasseroni, S. Mankovsky, J. Minár, H. Ebert, S. Moyerman, E. E. Fullerton, and F. Hellman, Phys. Rev. Lett. 109, 117201 (2012).

[39] S. Mankovsky, D. Ködderitzsch, G. Woltersdorf, and H. Ebert, Phys. Rev. B 87, 014430 (2013).

[40] E. Barati, M. Cinal, D. M. Edwards, and A. Umerski, Phys. Rev. B 90, 014420 (2014).

[41] C. Baldasseroni, C. Bordel, C. Antonakos, A. Scholl, K. H. Stone, J. B. Kortright, and F. Hellman, J. Phys.: Condens. Matter 27, 256001 (2015).

[42] M. Fähnle and D. Steiauf, Phys. Rev. B 73, 184427 (2006).

[43] K. Gilmore, Y. U. Idzerda, and M. D. Stiles, Phys. Rev. Lett. 99, 027204 (2007).

[44] A. Lodder, J. Phys. F 6, 1885 (1976).

[45] N. Papanikolaou, R. Zeller, P. H. Dederichs, and N. Stefanou, Phys. Rev. B 55, 4157 (1997).
[46] H. Böttger, Principles of the Theory of Lattice Dynamics (Akademie-Verlag, Berlin, 1983).

[47] K. Kreiner, H. Michor, G. Hilscher, N. Baranov, and S. Zemlyanski, J. Magn. Magn. Mater. 177-181, 581 (1998).

[48] E. F. Skelton and J. L. Katz, Phys. Rev. 171, 801 (1968).

[49] E. M. Gololobov, E. L. Mager, Z. V. Mezhevich, and L. K. Pan, Phys. Status Solidi B 119, K139 (1983).

[50] S. Mankovsky, K. Chadova, D. Ködderitzsch, J. Minár, H. Ebert, and W. Bensch, Phys. Rev. B 92, 144413 (2015).

[51] M. Jiang, X. Z. Chen, X. J. Zhou, Y. Y. Wang, F. Pan, and C. Song, J. Cryst. Growth 438, 19 (2016).

[52] Z. Celinski, K. Urquhart, and B. Heinrich, J. Magn. Magn. Mater. 166, 6 (1997).

[53] B. Heinrich, J. F. Cochran, and R. Hasegawa, J. Appl. Phys. 57, 3690 (1985).

[54] V. Kamberský, Phys. Rev. B 76, 134416 (2007).

[55] K. Gilmore, Y. U. Idzerda, and M. D. Stiles, J. Appl. Phys. 103, 07D303 (2008).

[56] X. Ke and G. J. Kramer, Phys. Rev. B 66, 184304 (2002).

[57] D. Steiauf and M. Fähnle, Phys. Rev. B 72, 064450 (2005). 